\title{
Aktuelles aus der Forschung
}

I. Kleiter ${ }^{1}$, M. S. Weber ${ }^{2}$

${ }^{1}$ Klinik für Neurologie, St. Josef-Hospital, Ruhr-Universität Bochum

${ }^{2}$ Institut für Neuropathologie und Klinik für Neurologie, Georg-August-Universität, Göttingen

\section{Stellenwert des MRT bei der Erfas- sung neuer Krankheitsparameter der MS}

Unter allen paraklinischen Parametern, die eingesetzt werden, um die Diagnose der Multiplen Sklerose (MS) zu stellen, aber auch um die individuelle Prognose abzuschätzen, hat das MRT im letzten Jahrzehnt zweifelsfrei am stärksten an Einfluss gewonnen. Am deutlichsten ist dies in den 2010 revidierten McDonaldDiagnosekriterien reflektiert; insbesondere die obligate Chronizität der Erkrankung wird in diesen zunehmend vereinfachten Kriterien nach immer kürzerer Erkrankungsdauer dokumentiert. Dies ist ohne Frage erstrebenswert, da die frühzeitige und damit effiziente Behandlung der Erkrankung für den individuellen Patienten von weitreichender Bedeutung ist. Grundsätzlich birgt diese Entwicklung allerdings den intrinsischen Nachteil einer möglichen Zunahme von Fehldiagnosen, welche konsekutiv im Kontext komplexer Therapiealgorithmen und immer effizienterer Therapeutika seltener infrage gestellt werden könnten. Um dieser Gefahr entgegenzuwirken, haben sich eine Reihe aktueller Publikationen zum Ziel gesetzt, zusätzliche und möglicherweise sogar spezifischere Krankheitsund Progressionsparameter der MS, wie z. B. die diffuse Gewebszerstörung in (otherwise) normal appearing white matter, die kortikale Läsionsbildung oder auch die Akkumulation leptomeningealer Entzündung, der MRT-Detektion zugänglich zu machen.

Den Stellenwert dieser neuen Entwicklungen fasst die im August 2015 veröffentlichte MAGNIMS (Magnetic resonance imaging in $\mathrm{MS}$ ) consensus guideline $\mathrm{zu}$ sammen [1]. Hier konnten wesentliche Strategien identifiziert werden, um bislang unterrepräsentierte Krankheitsmechanismen abzubilden. So könnte zukünftig beispielsweise die MR-basierte Spektroskopie den diffusen Gewebsschaden innerhalb des ZNS unabhängig von fokaler Läsionsbildung messen; in diesem $\mathrm{Zu}$ sammenhang konnte gezeigt werden, dass in Patienten mit einem klinisch iso- lierten Syndrom (CIS) in der nicht von der Läsion betroffenen Hemisphere NAzetylaspartat als Marker neuroaxonaler Schädigung erniedrigt und dagegen myoInositol als Marker glialer zellulärer Aktivierung erhöht war. Interessanterweise waren diese Veränderungen stärker ausgeprägt, wenn Patienten früh eine erneute Exazerbation im Sinne einer dann definitiven MS entwickelten. Weiterhin waren diese Veränderungen in Patienten mit ADEM und NMO nicht nachweisbar. Insofern könnte die Darstellung diffus entzündlicher Gewebsschädigungen zweifach genutzt werden; zum einen, um die diagnostische Spezifität des MRTs zu erhöhen, und zum anderen im Sinne eines frühzeitigen prognostischen Biomarkers. Weitere Parameter läsionsgebundener ZNS-Entzündung im Rahmen der MS wie z.B. die Ablagerung von Eisen oder auch der Nachweis einer Zentralvene in der Läsion könnten hinzutreten, um gemeinsam die Spezifität trotz eines sehr frühen Diagnosezeitpunkts zu gewährleisten.

Einen weiteren, bislang unterschätzten und in höchstem Maße behinderungsrelevanten Aspekt der MS-Pathologie dürfte die kortikale Läsionsbildung darstellen. Hierfür ist es entscheidend, die graue Substanz störungsfrei und unabhängig von Veränderungen der weißen Substanz sowie des Liquors abzubilden. Hierfür dürfte die sogenannte double inversion recovery (DIR) einen entscheidende Ansatz darstellen. Die Detektion in der MS offensichtlich sehr häufig auftretender kortikaler Läsionen wäre nicht nur zum Ausschluss von Differenzialdiagnosen extrem hilfreich, sondern könnte auch beitragen, um die geforderte Dissemination in Zeit und Ort läsionsgebundener Entzündung früher zu dokumentieren.

Zwei aktuelle Publikationen beschäftigen sich nun mit der pathophysiologischen Entwicklung dieser kortikalen Läsionen und mit der Frage, inwieweit die Anreicherung leptomeningealer Entzündung hierfür verantwortlich und im MRT abbildbar ist [2, 3]. Denn rezente neuropathologische Befunde legen nahe, dass die Aggregation meningealer Entzündungszellen in der Tat mit kortikaler Demyelini-

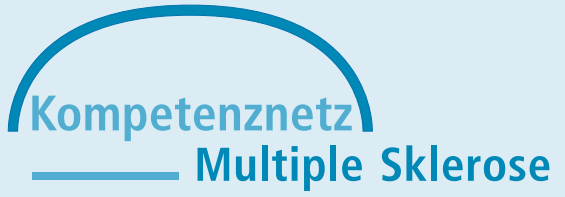

sierung und Läsionsbildung assoziiert sein könnte. Insofern könnte meningeale Entzündung sogar der entscheidende Trigger für die schubunabhängige schleichende Akkumulation körperlicher Behinderung darstellen. In beiden Publikationen wurde als Untersuchungstechnik die T2-gewichtete Fluid-attenuated inversion recovery (T2-Flair) angewendet. Diese zeigte nach Applikation von Kontrastmittel in $25 \%$ aller untersuchten Fälle eine leptomeningeale Anreicherung. In der weit überwiegenden Mehrzahl der Fälle wurde diese interessanterweise in einem einzelnen Sulcus nachgewiesen. Von entscheidender Bedeutung könnte sein, dass diese leptomeningeale Anreicherung in Patienten mit progredient verlaufender MS 1,7-fach häufiger zu finden war als in schubförmig-remittierender MS. Innerhalb der progredienten Verlaufsformen fand sich ein leptomeningeales Enhancement am häufigsten in primär progredienten MS-Fällen. Ein davon unabhängiger Zusammenhang mit Geschlecht, Alter oder Krankheitsdauer konnte nicht gefunden werden. In 2 dieser Patienten mit progressiver MS und in vivo Nachweis leptomeningealer Entzündung erfolgte im Verlauf eine Autopsie. Beide zeigten in den initial MRT-detektierten Entzündungsregionen ein deutliches perivaskuläres Infiltrat, das von T-Zellen, BZellen und Makrophagen dominiert wurde. In direkter Nachbarschaft dieses leptomeningealen Infiltrats fanden sich Gewebsabschnitte mit ausgedehnter kortikaler Demyelinisierung. Hiermit konnte die MRT-Detektierbarkeit leptomeningealer Entzündung bestätigt werden. Perspektivisch könnte der Nachweis leptomeningealer Entzündung also insbesondere bei progressiven MS-Formen, die im herkömmlichen MRT nur unzureichend zu monitorieren sind, einen wertvollen Aktivitätsmarker darstellen. Dies wäre zugleich ein erster Ansatz um in diesen untertherapierten Verlaufsformen mögliche Zielparameter therapeutischer Intervention zu entwickeln. Hierfür konnte in der zitierten Studie als interessanter Nebenaspekt dargestellt werden, dass in den untersuchten Fällen keines der heute zuge- 
lassenen und breit angewendeten MSTherapeutika einen Einfluss auf Auftreten oder Ausmaß leptomeningealer Entzündung hatten.

Zusammenfassend legen diese Studien also nahe, dass das MRT mit neuen Sequenzen und Strategien auch in Zukunft die entscheidende Methode bleiben wird, um in Patienten mit MS neben Schüben und fokaler Entzündung zusätzliche Krankheitsmechanismen wie diffusen ZNS-Gewebeschaden, kortikale Läsionsbildung oder chronisch-persistierende leptomeningeale Entzündung nachzuweisen. Diese Parameter könnten gewährleisten, dass die MRT-Untersuchung trotz immer früherer Diagnosestellung nicht an Spezifität verliert, und zugleich genutzt werden, um unsere komplexen Therapiealgorithmen mit zusätzlichen, prognostisch behinderungsrelevanten MRT-Parametern zu flankieren.

Erklären Schwankungen im Melatoninspiegel saisonale Unterschiede der MS-Krankheitsaktivität?

$\nabla$

Seit Langem ist bekannt, dass MS-Schübe im Herbst und beginnenden Winter seltener als im Frühjahr und Frühsommer auftreten. Kürzlich konnte zudem die Breitengradabhängigkeit dieses Effekts gezeigt werden [4]. Die biologische Grundlage der saisonalen Häufung ist unklar, vermutet wurde ein Zusammenhang mit der Sonneneinstrahlung, dem Vitamin-DSpiegel und mit viralen Infekten.

Farez, Correale und Kollegen präsentieren nun in einer aktuellen, in der renommierten Zeitschrift Cell erschienenen Arbeit einen möglichen Erklärungsmechanismus für die verminderte Erkrankungsaktivität im Herbst und den Wintermonaten [5]. Sie fanden bei 139 argentinischen Patienten mit schubförmiger MS eine Abhängigkeit der Schubrate vom Melatoninspiegel. War dieser erhöht, wie es für die dunklen Jahreszeiten typisch ist, traten etwa ein Drittel weniger Schübe auf als bei niedrigem Melatoninspiegel in den hellen Monaten. Dagegen korrelierten weder der Vitamin-D-Spiegel, noch die Häufigkeit respiratorischer Infektionen und das Ausmaß der UV-Strahlung mit der Schubrate. Um den zugrunde liegenden Mechanismus aufzudecken, wurden In-vitro-Differenzierungsassays von humanen und murinen T-Zellen durchgeführt, die intrazelluläre Signaltransduktion in T-Zellen untersucht und Mäuse im Tiermodell der experimentellen autoimmunen Enzephalo- myelitis mit Melatonin behandelt. Hier zeigte sich, dass die Gabe von Melatonin zu einem abgeschwächten Krankheitsverlauf und verringerten Zahl pathogener Th17-Zellen sowie zu eine Abnahme der Antigen-spezifischen T-Zell-Proliferation führt. Darüber hinaus fand sich unter Melatoninbehandlung im murinen wie humanen In-vitro-System ein Anstieg von IL-10-produzierenden T-Zellen, sogenannten regulatorischen $\operatorname{Tr} 1-Z e l l e n$. Auch die verminderte Th17-Differenzierung unter Melatonin konnte in humanen TZellen repliziert werden.

Melatonin bindet an den Membranrezeptor MTNR1A und den nukleären Rezeptor ROR- $\alpha$. Wie die Autoren mit einer Reihe von Experimenten zeigen konnten, wird die Suppression von Th17-Zellen über MTNR1A und die intrazellulären Signalproteine Erk1/2, C/EBP $\alpha$ und NFIL3 vermittelt. In der Endstrecke führte eine Aktivierung dieser Signalproteine zu einer Hemmung des Th17-spezifischen Tran-

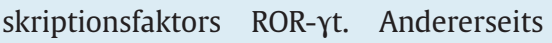
führte eine Melatoninbehandlung über den Erk-Signalweg zu einer Aktivierung von ROR- $\alpha$ und Tr1-assoziierter Moleküle und verstärkte die suppressiven Eigenschaften von Tr1-Zellen.

Sowohl die In-vitro-Effekte auf die T-ZellDifferenzierung wie die In-vivo-Wirksamkeit von Melatonin im Modell der experimentellen autoimmunen Enzephalomyelitis wurden unabhängig von einer anderen Gruppe beschrieben [6]. Zudem fand sich in einer Untersuchung von 590 MS-Patienten und Kontrollen aus Finnland eine Assoziation von SNPs in Genen des Melatonin-Signalwegs mit progredienten MS-Krankheitsverläufen [7]. Der Zusammenhang zwischen Melatoninspiegel und saisonaler Häufung von MS-Schüben wurde bereits 1993 von Sandyk und Awerbuch postuliert [8], was einmal mehr zeigt, dass eine alte Idee mit gewisser Latenz erneut aufgenommen werden kann. Sandyk und Awerbuch stellten auch die interessante, jedoch noch zu überprüfende Hypothese auf, dass die häufige Erstmanifestation der MS im jungen Erwachsenenalter durch den bis zur Pubertät stark abfallenden Melatoninspiegel erklärt werden könnte. Passend hierzu zeigten nachfolgende Studien dieser Autoren und mehrere aktuelle Studien aus Israel und dem Iran erniedrigte Melatoninspiegel bei MS-Patienten, insbesondere bei längerem Erkrankungsverlauf. Auch wenn durch diese Befunde nahegelegt wird, dass erhöhte Melatoninspiegel mit einer niedrigeren MS-Erkrankungsak- tivität assoziiert sind, bleibt unklar ob ein ähnlicher Zusammenhang mit der Inzidenz der MS besteht. Zumindest Befunde aus Skandinavien sprechen dagegen, da die MS-Inzidenz dort trotz der langen Phasen der Dunkelheit gegenüber südeuropäischen Ländern erhöht ist.

Der in der referierten Studie postulierte Zusammenhang zwischen jahreszeitlicher Sonneneinstrahlung, Melatoninspiegel, TZell-Antworten und MS-Erkrankungsaktivität muss in unabhängigen Studien mit größerer Teilnehmerzahl bestätigt werden. Insbesondere die zugrunde liegenden immunologischen Mechanismen sind noch unzureichend verstanden, daher ist zum jetzigen Zeitpunkt vor individuellen Heilversuchen mit Melatonin oder der chemisch ähnlichen Substanz Agomelatin zu warnen.

\section{Literatur}

1 Rovira A, Wattjes MP, Tintoré $M$ et al. Evidence-based guidelines: MAGNIMS consensus guidelines on the use of MRI in multiple sclerosis-clinical implementation in the diagnostic process. Nat Rev Neurol 2015; 11: 471-482

2 Absinta M, Vuolo L, Rao A et al. Gadoliniumbased MRI characterization of leptomeningeal inflammation in multiple sclerosis. Neurology 2015; 85: 18-28

3 Eisele P, Griebe M, Szabo K et al. Investigation of leptomeningeal enhancement in MS: a postcontrast FLAIR MRI study. Neurology 2015; 84: 770-775

4 Spelman T, Gray O, Trojano M et al. Seasonal variation of relapse rate in multiple sclerosis is latitude dependent. Ann Neurol 2014; 76 : $880-890$

5 Farez MF, Mascanfroni ID, Méndez-Huergo SP et al. Melatonin contributes to the seasonality of multiple sclerosis relapses. Cell 2015; 162: $1338-1352$

6 Alvarez-Sanchez N, Cruz-Chamorro I, LópezGonzález A et al. Melatonin controls experimental autoimmune encephalomyelitis by altering the $\mathrm{T}$ effector/regulatory balance. Brain Behav Immun 2015: Epub ahead of print; doi: 10.1016/j.bbi. 2015.06.021

7 Natarajan R, Einarsdottir E, Riutta A et al. Melatonin pathway genes are associated with progressive subtypes and disability status in multiple sclerosis among Finnish patients. J Neuroimmunol 2012; 250: 106 110

8 Sandyk R, Awerbuch G. Multiple sclerosis: relationship between seasonal variations of relapse and age of onset. Int J Neurosci 1993; 71: $147-157$

\section{Korrespondenzadresse}

Krankheitsbezogenes Kompetenznetz Multiple Sklerose

Klinikum rechts der Isar der TU München Einsteinstraße 1, 81675 München info@kkn-ms.de www.kompetenznetz-multiplesklerose. de 